\title{
Robust Integration of Motion Information in the Fly Visual System Revealed by Single Cell Photoablation
}

\author{
Julia Kalb, Martin Egelhaaf, and Rafael Kurtz \\ Department of Neurobiology, University of Bielefeld, D-33501 Bielefeld, Germany
}

\begin{abstract}
In the brain, sensory information needs often to be read out from the ensemble activity of presynaptic neurons. In the most basic case, this may be accomplished by an individual postsynaptic neuron. In the visual system of the blowfly, an identified motion-sensitive spiking neuron is known to be postsynaptic to an ensemble of graded-potential presynaptic input elements. Both the presynaptic and postsynaptic neurons were shown previously to be capable of representing the velocity of preferred-direction motion reliably and linearly over a large frequency range of velocity fluctuations. Accordingly, the synaptic transfer properties of the connecting excitatory synapses between individual input elements and the postsynaptic neuron were shown to be linear over a similar range of presynaptic membrane potential fluctuations. It was not known, however, how the postsynaptic neuron integrates and reads out the presynaptic ensemble activity. We were able to compare the response properties of the integrating cell before and after eliminating individual presynaptic elements by a laser ablation technique. For most of the input elements, we found that their elimination strongly affected the activity of the postsynaptic neuron but did not degrade its performance to encode motion with constant and time-varying velocity. Our results suggest that the integration of individual synaptic inputs within the neural circuit operates with some redundancy. This feature might help the postsynaptic neuron to encode in a highly robust way the direction and the velocity of self-motion of the animal.
\end{abstract}

Key words: visual motion; synaptic transmission; invertebrates; sensory neurons; sensory integration; photoablation

\section{Introduction}

The high degree of convergence in sensory and neural systems is often based on synaptic integration of inputs that provide very similar information. It is the task of synaptic filtering and postsynaptic integration to extract relevant information from the incoming signals. Examples of how this is done and how either linear combination of inputs or nonlinear synaptic integration is beneficial have been provided in numerous species and brain regions. A prominent example is noise reduction during integration of photoreceptor signals in vertebrates and invertebrates (Juusola et al., 1995; de Ruyter van Steveninck and Laughlin, 1996; Field and Rieke, 2002).

Here we address the computational consequences of synaptic convergence of sensory neurons at a higher integration level of the nervous system. We address in the third visual neuropil of the blowfly the question of how synaptic signals are integrated at excitatory synaptic connections between a small group of identified presynaptic neurons and one postsynaptic neuron. The blowfly visual system is particularly suitable for this sort of analysis because the cellular basis of neuronal computation can be studied in vivo, using the intact sensory pathways to evoke central

Received Jan. 23, 2006; revised May 29, 2006; accepted June 18, 2006.

This work was supported by the Deutsche Forschungsgemeinschaft. We thank Dr. Holger Krapp and three anonymous reviewers for helpful comments on a previous version of this manuscript.

Correspondence should be addressed to Julia Kalb, Department of Neurobiology, University of Bielefeld, Universitätsstraße 25, Postfach 1001 31, D-33501 Bielefeld, Germany. E-mail: julia.kalb@uni-bielefeld.de. D0I:10.1523/JNEUROSCI.1327-06.2006

Copyright $\odot 2006$ Society for Neuroscience $\quad$ 0270-6474/06/267898-09\$15.00/0 synaptic activity by visual stimulation (for review, see Egelhaaf and Borst, 1993; Borst and Haag, 2002; Egelhaaf et al., 2002).

The analyzed neurons belong to a population of $\sim 60$ visual motion-sensitive tangential cells in the fly visual neuropil. These neurons are tuned to sense particular patterns of retinal image flow as is induced during self-motion of the animal (for review, see Hausen and Egelhaaf, 1989; Borst and Haag, 2002). The presynaptic VS cells belong to a group of 10 motion-sensitive neurons that possess large receptive fields and respond predominantly to vertical motion by graded depolarization or hyperpolarizations even in their axon terminals (Hengstenberg, 1982; Hengstenberg et al., 1982; Krapp et al., 1998). The postsynaptic V1 cell integrates the motion signals from the presynaptic VS cells, transforms them into spike activity, and conveys the motion information to the contralateral visual system (Kurtz et al., 2001; Warzecha et al., 2003). Both the presynaptic and postsynaptic neurons were shown previously to encode preferred-direction velocity fluctuations linearly and reliably over a frequency range of up to $\sim 10 \mathrm{~Hz}$ (Warzecha et al., 2003). Accordingly, the overall synaptic transfer between VS cells and V1 was shown to operate almost linearly over a wide range of presynaptic membrane potential fluctuations (Kurtz et al., 2001; Warzecha et al., 2003). However, in the previous experiments, the presynaptic VS cells could not be stimulated individually. This is inevitable when using visual motion stimulation, because the receptive fields of the presynaptic neurons overlap to a large extent (Krapp et al., 1998; Warzecha et al., 2003). Consequently, our previous studies did not allow us to assess the contribution of individual VS cells to the performance of the postsynaptic V1 cell. To characterize the contribution of individual inputs during syn- 
aptic integration of visual motion signals, we eliminated by laser ablation single VS cells and studied the functional consequences for the performance of $\mathrm{V} 1$ in extracting motion information from the activity of the remaining VS cells.

\section{Materials and Methods}

Animal preparation and electrophysiology. Preparations of 1- to 2-d-old female blowflies (Calliphora vicina) bred in our laboratory culture followed Dürr and Egelhaaf (1999) with the modification that both optic lobes were exposed. The head of the animal was aligned with the monitor used for visual stimulation by adjusting it according to the symmetrical deep pseudopupil in the frontal region of both eyes. Experiments were performed at temperatures ranging from 20 to $25^{\circ} \mathrm{C}$. V1 spike activity was recorded extracellularly from its output arborizations in the left optic lobe (see Fig. 1). V1 could be identified by its excitatory responses to downward motion in the contralateral frontolateral visual field (Krapp et al., 2001). Extracellular recordings were done with glass electrodes [GC150TF-10 (Clark Electromedical, Edenbridge, UK); electrode resistances, 4-8 M $\Omega$ when filled with $1 \mathrm{M} \mathrm{KCl}$ ) pulled on a GMZ Universal puller (Zeitz, Augsburg, Germany). Spikes were thresholded, and the resulting pulses were sampled at $5 \mathrm{kHz}$ and analog-to-digital converted (DT 3001; Data Translation, Marlboro, MA). After a stable V1 recording was established, a presynaptic VS cell was penetrated close to its output region in the right optic lobe (see Fig. 1). VS cells could be identified by graded depolarizations during downward motion in the ipsilateral visual field (Krapp et al., 1998). Intracellular glass electrodes (GC100TF-10; Clark Electromedical) were pulled on a Brown Flaming puller (P97; Sutter Instruments, San Rafael, CA). The electrode tips were filled with a saturated solution of the phototoxic fluorescent dye 6-carboxyfluorescein (Invitrogen, Carlsbad, CA) in $1 \mathrm{~m}$ potassium acetate, and the shaft of the electrode was filled with $1 \mathrm{M} \mathrm{KCl}$. The electrodes had resistances between 40 and $80 \mathrm{M} \Omega$.

Visual stimulation. Our stimuli consisted of square-wave gratings (spatial wavelength, $12^{\circ}$; mean luminance, $15.6 \mathrm{~cd} / \mathrm{m}^{2}$ ), which were generated by a personal computer-controlled image synthesizer (Picasso; Innisfree, Cambridge, MA) and displayed on a cathode ray tube (model 608; Tektronix, Wilsonville, OR) at a frame rate of $183 \mathrm{~Hz}$. The center of the monitor screen was at an azimuth/elevation of $55^{\circ} / 26^{\circ}$, with $0^{\circ}$ corresponding to the frontal midline of the animal. The horizontal extent of the stimulus pattern was $90^{\circ}$ and the vertical extent was $110^{\circ}$.

The pattern was moved either at constant or randomly fluctuating velocities. We used four different constant velocities $(24,37,74$, and $96 \%$ ) corresponding to temporal frequencies of 2, 3, 6, and $8 \mathrm{~Hz}$. The presentation of each constant-velocity stimulus lasted $1.9 \mathrm{~s}$ and was followed by an interval of $1.7 \mathrm{~s}$, during which the pattern was not moving. For dynamic velocity stimulation, we generated eight different low-passfiltered Gaussian white-noise velocity fluctuations with an SD of $100 \%$ and an average velocity of $0 \%$ s. The dynamic motion presentations lasted for $2.45 \mathrm{~s}$. Repeated presentations were separated by intervals of $1.5 \mathrm{~s}$ and were provided in a pseudorandom order. The brightness contrast of the motion stimuli was 0.54 .

Laser ablation. During simultaneous V1/VS dual recordings, single presynaptic VS cells were filled with the phototoxic fluorescent dye 6-carboxyfluorescein in the electrode tip by applying tonic hyperpolarizing current (3.5-8 nA, 5-15 min). To selectively kill the dye-filled VS cells, we used a fiber-coupled blue laser module ( $488 \mathrm{~nm}$; FTEC488; Blue Sky Research, Milipitas, CA). The optical fiber (125 $\mu \mathrm{m})$ of the laser was positioned close to the dye-filled neuron. We operated the laser module at the maximum power of $35 \mathrm{~mW}$, which corresponded to an output power of $\sim 26 \mathrm{~mW}$ at the end of the fiber.

We performed control experiments to document the laser-induced effects on the ablated VS cells (supplemental Figs. 1, 2, available at www. jneurosci.org as supplemental material). In accordance with Farrow et al. $(2003,2005)$, the visual responsiveness of the ablated cells was entirely abolished. Moreover, before laser ablation, dye-filled neurons were clearly visible by fluorescence microscopy (for details on methods, see Kurtz, 2004) but vanished after laser illumination. Because the efficiency and nature of the ablation procedure on the same neuronal cell class has been thoroughly investigated previously (Farrow et al., 2003), we performed these controls only in a small subset of cells. The specificity of laser ablation for the dye-filled neurons was tested by filling neurons that are not presynaptic to $\mathrm{V} 1$ and monitoring motion responses of the V1 before and after laser ablation of these neurons (see Results).

Data analysis. Data were analyzed off-line with Matlab 6.5 (MathWorks, Natick, MA). Spike activity histograms were obtained to compare the responses of V1 before and after ablation of a presynaptic VS cell. The spikes of V1 were counted within $100 \mathrm{~ms}$ time windows and averaged over consecutive motion presentations. All data are given as means and corresponding SDs.

The coherence was determined to analyze the impact of ablation on the performance of V1 in encoding white-noise motion-velocity fluctuations. The coherence was used as a measure of how well the time-varying motion velocities of the stimulus can be reconstructed from the activity of V1. It is based on a linear filter, which, when convolved with the activity of V1, minimizes the error between reconstructed and actual motion velocities. The coherence calculates to what extent the frequency components of the time-dependent signals can be transformed linearly and reliably into one another. If in the frequency range the coherence equals 1, the relationship between the signals is linear and noise free, whereas a coherence value $<1$ is attributed to noise and/or nonlinearities in the system.

To calculate the coherence, the eight dynamic motion sequences and the corresponding V1 responses were Fourier transformed, and the autocorrelations and cross-correlations were calculated as the products of the corresponding complex functions. The coherence function is given by the following (for review, see Borst and Theunissen, 1999):

$$
\begin{aligned}
\gamma^{2}(f)=\left(\left\langle R_{i}^{*}(f) \cdot S_{i}(f)\right\rangle \cdot\right. & \left.\left\langle S_{i}^{*}(f) \cdot R_{i}(f)\right\rangle\right) / \\
& \left(\left\langle S_{i}(f) \cdot S_{i}^{*}(f)\right\rangle \cdot\left\langle R_{i}(f) \cdot R_{i}^{*}(f)\right\rangle\right) .
\end{aligned}
$$

To compare the best linear filters used to reconstruct the motion-velocity trace before and after ablation, the filters were determined in the frequency domain by the following formula:

$$
H(f)=\left(\left\langle R_{i}^{*}(f) \cdot S_{i}(f)\right\rangle\right) /\left(\left\langle R_{i}(f) \cdot R_{i}^{*}(f)\right\rangle\right) .
$$

We reconstructed the motion-velocity traces by convolving the spike responses of $\mathrm{V} 1$ with the impulse response of the best linear filter obtained by reverse Fourier transformation of $H(f)$. The brackets denote averages over time segments of motion sequences $\left(S_{i}\right)$ and corresponding neural responses $\left(R_{i}\right)$, $\cdot$ denotes the dot product, and ${ }^{\star}$ denotes the complex conjugate. Each of the eight motion stimuli and the neural responses were divided into three time segments. For the analyses, the neural responses and the visual stimulus were binned to $5 \mathrm{~ms}$ time windows. The eight dynamic motion traces were presented four times in each experiment.

The assessment of valid coherences is complicated by the inevitably limited number and duration of dynamic motion traces. Thus, particularly in the lower-frequency range, ablation-induced decreases of coherence values might be masked by noise. To exclude this possibility, we demonstrated the validity of our results on the basis of an extended set of V1 responses $(n=10$ presentations of each dynamic motion trace; the experiment was done on $n=5$ cells). Over the entire frequency range, these control experiments yielded similar coherence values as obtained with the smaller number of presentations (see average coherences in Fig. $3 B$ ). As a second control, a dynamic motion sequence ( $n=25$ presentations of the motion sequence, $n=8$ experiments) was used that lasted $5.12 \mathrm{~s}$ and that, therefore, provided a more reliable estimation of coherence values for low frequencies (see Fig. $3 B$ ). With this longer dynamic motion sequence, similar coherence values were obtained as under our standard conditions. Because of the limited duration of stable recordings, we performed the control experiments without simultaneously ablating presynaptic cells.

We determined to what extent the information content of the V1 responses changed after ablation of a VS cell. We applied a measure that 
calculates the information without assuming a linear relationship between stimulus and responses (Brenner et al., 2000):

$$
I(\text { spike })=\frac{1}{T} \int_{0}^{T}\left(\frac{r(t)}{\bar{r}}\right) \log _{2}\left(\frac{r(t)}{\bar{r}}\right) .
$$

Here, $r(t)$ is the time-dependent spike activity, $\bar{r}$ is the time average of the spike activity, and $T$ is the length of the response segment. Equation 3 provides the information per spike from the spike-frequency histogram and the mean spike activity of the responses to a stimulus, thus ignoring higher-order structure in the spike train. The information per spike multiplied with the spike activity during each dynamic motion sequence yields the information rate.

Because of the limited dataset from our ablation experiments, the time-dependent spike activity might be contaminated by noise. Taking advantage of the distinct frequency distributions of noise and signal in tangential cells (Warzecha et al., 2003), the time averages of spike activities were low-pass filtered with a cutoff frequency of $10 \mathrm{~Hz}$ to prevent an overestimation of calculated information rates. As a control, the same analysis was done on a substantially larger dataset. Then the dataset was reduced to the same size as in our experiments and was quantified in an identical way. The analysis yielded similar estimates of information rates for the reduced and the complete dataset. The average information rate for the reduced dataset was $42 \pm 7 \mathrm{bits} / \mathrm{s}$ compared with $40 \pm 5 \mathrm{bits} / \mathrm{s}$ for the complete dataset (mean $\pm \mathrm{SD} ; n=4)$.

\section{Results}

We investigated the contribution of individual synaptic motion inputs to the overall postsynaptic V1 activity. We compared in vivo the response properties of the postsynaptic V1 cell during motion stimulation before and after eliminating by laser ablation one of its presynaptic input elements. Figure 1 shows a schematic of the motion pathway with the V1 cell and one of the presynaptic VS cells.

VS cells respond to downward motion with depolarizing shifts of their membrane potential even when recorded close to their output terminals. VS cells receive their motion sensitivity by many small-field retinotopic motion-sensitive elements that are pooled by their extensive dendritic tree. Because the dendrites of the different VS cells are slightly displaced with respect to each other, their receptive fields cover neighboring but overlapping areas in the visual field (Hengstenberg, 1982; Hengstenberg et al., 1982; Krapp et al., 1998). Moreover, neighboring VS cells were concluded to be electrically coupled, thereby increasing their receptive fields beyond their retinotopic input area (Farrow et al., 2005). The coupling strength between VS cells was demonstrated to be fairly weak: current injections of up to $10 \mathrm{nA}$ into a VS cell only elicited a depolarization of $\sim 1 \mathrm{mV}$ in the nearest neighbor (Haag and Borst, 2004).

V1 integrates the motion signals from presynaptic VS cells and transforms them into spike activity. Simultaneous VS/V1 recordings (Kurtz et al., 2001; Warzecha et al., 2003) and the comparison of the receptive field of V1 with that of the VS cells (Krapp et al., 1998, 2001) suggests that V1 gets strong input from VS1-VS3 and weak input from one or several of the VS cells with more lateral sensitivities to downward motion: the receptive field of V1 corresponds well to a combination of the receptive fields of the contralateral VS1-VS3. A minor contribution of VS4 and potentially also of VS5 and VS6 to the response properties of V1 is suggested by an examination of its lateral receptive field.
We identified VS cells by their preferred directions and the location of their receptive fields in the frontal visual field (Krapp et al., 1998). Moreover, synaptic connection to the postsynaptic V1 cell was established by injecting positive or negative current into the VS cell under investigation and by observing increases and decreases in spike activity of V1. Because VS2 and VS3 have almost identical receptive field structures and very similar functional properties, we did not distinguish between them throughout this study. In contrast, we could easily identify VS1 by its sensitivity to back-to-front horizontal motion in its dorsolateral receptive field. We can distinguish VS4-VS6 from VS1-VS3 by their more lateral maximum sensitivity to vertical downward motion, but we cannot differentiate individual cells within each group of VS cells with absolute certainty.

We determined the motion-induced spike activity of V1 during visual stimulation with a drifting square-wave grating (Fig. 2). The pattern covered the major part of the receptive field of $\mathrm{V} 1$ and moves downward, i.e., in the preferred direction of V1, at four different constant velocities corresponding to temporal frequencies of 2, 3, 6, and $8 \mathrm{~Hz}$. Temporal frequency is defined as the angular velocity of the pattern divided by its spatial wavelength. Because the spatial wavelength in our experiments is constant, higher frequencies correspond to higher velocities of pattern motion.

\section{Impact of VS2/3 cell ablation on the responses of V1 to constant-velocity motion}

During simultaneous recordings of a VS2/3 cell and V1, the presynaptic VS2/3 cell was filled with a phototoxic dye. Figure $2 \mathrm{~A}$ shows a sample of spike trains indicating the occurrence of V1 action potentials in sections of individual stimulus presentations as well as spike-frequency histograms of V1 before and after ablation of the presynaptic VS2/3 neuron. Pattern motion is indicated by the bars. Individual $1.9 \mathrm{~s}$ presentations of motion with various temporal frequencies are separated by 1.7 s intervals, during which the pattern was stationary. The activities were evaluated within $100 \mathrm{~ms}$ time windows and averaged over individual traces $(n=4)$ both before and $40 \mathrm{~min}$ after ablation of a VS2/3 neuron. Before ablation, V1 shows a moderate baseline activity of $19.4 \pm 13.7$ spikes/s and is strongly activated during visual motion. V1 reaches slightly different stimulus-induced response levels for the different motion velocities. The activity of V1 rises highest at a temporal frequency of $6 \mathrm{~Hz}$ with peak values of $221 \pm$ 11.6 spikes/s. At the lower temporal frequencies, the response amplitude remains nearly constant over the entire period of mo- 

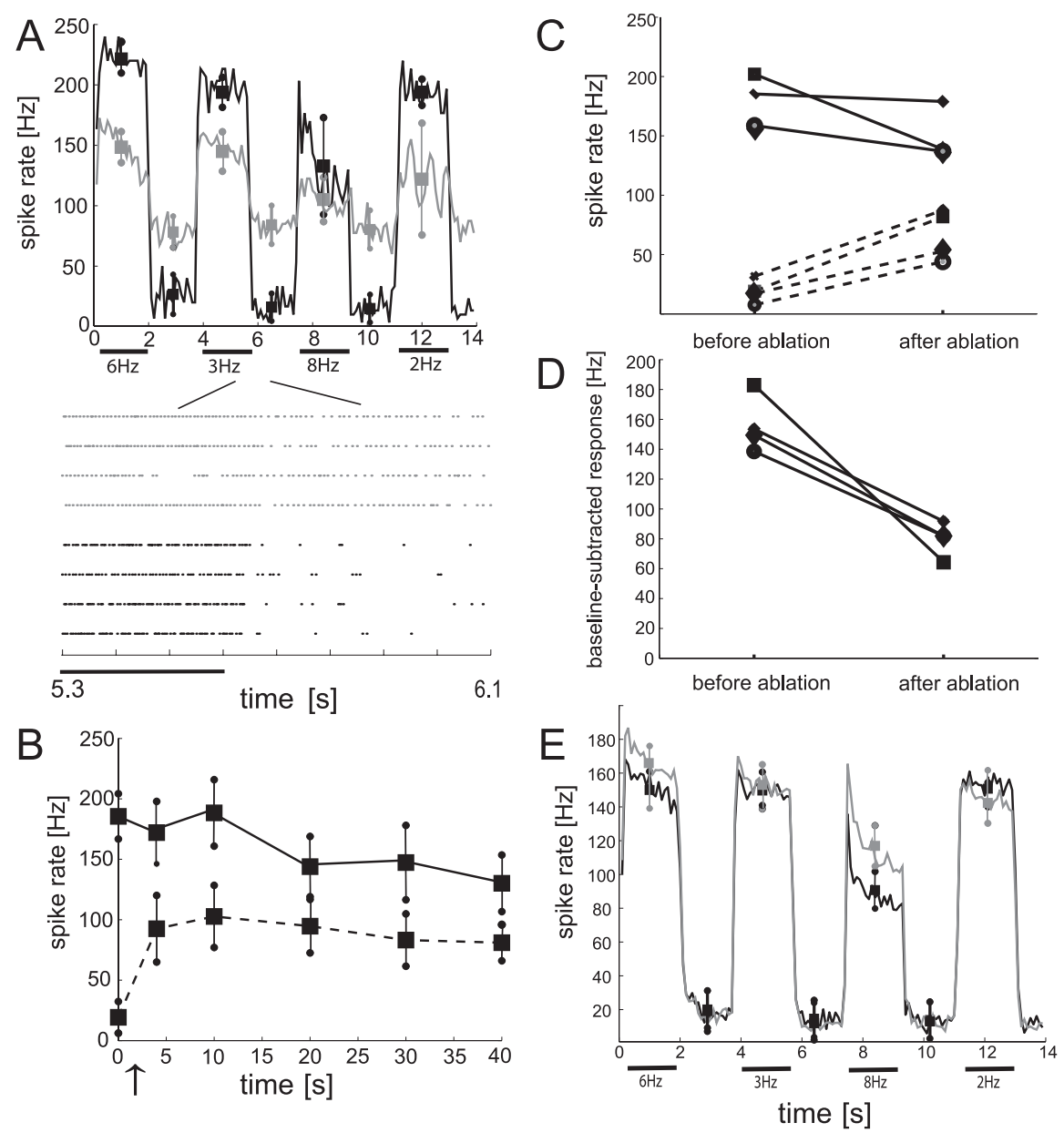

Figure 2. Changes in the response characteristic of $\mathrm{V} 1$ during constant-velocity motion stimulation before and after selective ablation of a VS2/3 cell. A, Top, Average response amplitudes of a sample V1 before (black line) and $40 \mathrm{~min}$ after (gray line) ablation of a VS2/3 cell. Black bars indicate the pattern motion. The pattern moved with four different velocities corresponding to the indicated temporal frequencies. Each motion presentation lasted 1.9 s separated by a 1.7 s interval with a stationary pattern. Response amplitudes were binned into $100 \mathrm{~ms}$ time windows and averaged over four stimulus repetitions. Squares and circles denote means and SDs of motion-induced and baseline activity of $\mathrm{V} 1$, respectively. Motion-induced means and SDs were determined $100 \mathrm{~ms}$ after motion onset within $1.5 \mathrm{~s}$ windows. Correspondingly, we calculated the means of baseline activity in $1.5 \mathrm{~ms}$ windows starting $100 \mathrm{~ms}$ after the cessation of each motion presentation. Bottom, Raster plots of the corresponding responses before (black dots) and after (gray dots) ablation. Each dot denotes the occurrence of a V1 spike. $\boldsymbol{B}$, Time course of both the average baseline activity (data points connected by the dashed line) and the motion-induced activity (data points connected by the solid line) of V1 relative to the time of VS2/3 ablation. The arrow indicates the time of ablation. Error bars indicate SD over four individual traces. The average responses were calculated in the time windows as specified in A. C, Summarized changes in average baseline (dashed lines) and motion-induced (solid lines) activity of V1 after VS2/3 ablation. The data are based on four ablation experiments with individual experiments indicated by different symbols. The cell shown in $A$ and $B$ corresponds to the squares. Data after ablation were gathered from comparable time windows after the ablation procedure $(10-20 \mathrm{~min}$ after ablation). The motion-induced responses (solid lines), averaged over the 2, 3, and $6 \mathrm{~Hz}$ temporal frequency conditions, and baseline (dashed lines) response amplitudes before and after ablation, each presented four times. After ablation, the baseline activity increased with an overall factor of $3.9 \pm 1$.4. The motion-induced response was weakened after ablation in all experiments, although to a different extent. $\boldsymbol{D}$, For the same experiments, the average baseline activity was subtracted from the corresponding average motion-induced response amplitudes. VS2/3 ablation always led to a compressed response range of $\mathrm{V} 1$ for the representation of preferred-direction motion. $\boldsymbol{E}$, Control experiments. Average spike-frequency histograms obtained from 10 different $\mathrm{V} 1$ recordings during constant-velocity motion stimulation before (black line) and after (gray line) laser illumination without previous dye filling of a presynaptic VS cell. Squares and circles indicate corresponding means and SDs of V1 spike activities.

tion stimulation. Only at $8 \mathrm{~Hz}$ can a pronounced initial response transient and a decline to a lower steady-state value be observed.

After ablation of VS2/3 by high-intensity laser illumination, two prominent changes emerge in the response characteristic of $\mathrm{V} 1$ : we observe a strong rise in the mean baseline activity of $\mathrm{V} 1$ to, on average, four times the preablation level $(81 \pm 15$ spikes/s after ablation) and a drop in the motion-induced response ampli- tudes. This drop is qualitatively consistent for all temporal frequencies of the moving pattern and ranges from $21 \%$ at $8 \mathrm{~Hz}$ to $36 \%$ at $2 \mathrm{~Hz}$. We monitored the $\mathrm{V} 1$ responses for up to $1.5 \mathrm{~h}$ after ablating VS2/3 and found the ablation-induced response changes to be persistent over time (Fig. 2B).

Together, the rise in baseline activity and the decrease in motioninduced activity lead to a strong reduction in the response range that V1 can exploit to signal different preferred-direction motion velocities. This compression of the preferred-direction response range approaches $70 \%$, with average values of 186 spikes/s before ablation compared with 58 spikes/s after ablation (mean motion-induced activity with corresponding baseline activities subtracted). Because our stimulus protocol includes no null-direction motion, we cannot exclude that the compressed response range for preferred direction is compensated for by an increase of the response range for null-direction motion.

To test whether these ablationinduced changes in the response properties of V1 are a genuine consequence of the ablation procedure rather than just an unspecific effect of laser illumination, we performed control experiments and illuminated the preparation by the laser beam without previous loading one of the VS cells with the phototoxic dye. These control experiments validate the specificity of the above depicted ablation-induced effects. We find neither persistent increases in baseline activity nor strong changes in response amplitudes of individual V1 cells $(n=10)$ (Fig. $2 E$ ) attributable to laser illumination without previous dye-filling of presynaptic VS cells. If at all, response amplitudes are slightly raised after control laser illumination, at least in the conditions with fast pattern motion. Rather than to laser illumination, this effect may be attributable to general variability in the activity of V1, which we found to be higher during fast than during slow pattern velocities. In some of these control experiments, tangential neurons that are not synaptically coupled to V1 were dye filled and ablated to rule out unspecific effects that may potentially be caused by phototoxic conversion products of the dye spilled out from ablated neurons.

We could reproduce similar ablation-induced rises in the baseline activity of $\mathrm{V} 1$, as shown in Figure 2, $A$ and $B$, for one 
example, in all experiments in which we killed a VS2/3 cell $(n=4)$. However, the impact of ablation on the activity of V1 is often less prominent than for the sample cell shown in Figure 2. Figure 2, $C$ and $D$, summarizes the changes in average baseline and motion-induced activity of $\mathrm{V} 1$ after ablation of VS2/3: in all experiments, the baseline activity increased by $>50 \%$ of its preablation value (average \pm SD rise factor, $3.9 \pm 1.4$ ). However, because we observe in none of the experiments an increase of the motion-induced response amplitudes of V1 after ablation of VS2/3, the increment in baseline activity always leads to a compressed preferred-direction response range of V1. Figure $2 D$ shows the baseline-subtracted response amplitudes. The compression ranges from 67 to $88 \%$ relative to the response range before ablation.

Because the connections between V1 and its input elements are formed by excitatory synapses (Kurtz et al., 2001; Warzecha et al., 2003), the increased baseline activity cannot be attributed to a loss of inhibitory input. Ablated cells become leaky, as is indicated by an increase in conductance (Farrow et al., 2003) and leakage of fluorescence-dye (supplemental Fig. 1, available at www.jneurosci.org as supplemental material), and strongly depolarize (Farrow et al., 2003). Therefore, the sustained increase in baseline activity is most likely attributable to an increase in excitation (see Discussion). Thus, after ablation of VS2/3, the activity range of the postsynaptic $\mathrm{V} 1$ cell appears to be shifted from its normal to a significantly higher activity level at rest.

Next we investigated the functional implications of the ablation-induced changes in response properties of $\mathrm{V} 1$ for the ability of the cell to encode time-dependent fluctuations in motion velocity.

\section{Impact of VS2/3 cell ablation on the performance of $\mathrm{V} 1$ to encode time- dependent motion velocity}

Synaptic transfer of time-varying motion information between VS cells and V1 was shown to be reliable and linear for visually elicited graded presynaptic membrane potential fluctuations of up to $10 \mathrm{~Hz}$ (Warzecha et al., 2003). Because the receptive fields of VS cells overlap to a large extent, it is not possible to exclusively activate an individual presynaptic VS cell by visual stimulation. Thus, an analysis of synaptic transfer that is based on visual stimulation of the intact neuronal circuit does not allow us to dissociate the contribution of individual presynaptic VS cells to the coding properties of V1. Therefore, we ablated single VS cells and subsequently compared the ability of $\mathrm{V} 1$ to read out time-dependent motion information from the activity of the remaining VS cells with that of the intact ensemble.

To characterize the ability of V1 to encode motion velocity, we generated eight different stimuli consisting of band-limited white-noise velocity fluctuations. The pattern moved randomly in either the preferred or null direction. Figure $3 \mathrm{~A}$ displays one of these dynamic motion traces of $2.45 \mathrm{~s}$ duration, followed by a $1.5 \mathrm{~s}$ interval during which the pattern was not moving (gray line). The spike histogram before ablation of a VS2/3 cell (black line) shows the spike activity of V1 during stimulus presentation. The motion-induced responses strongly modulate with pattern velocity: depending on the instantaneous pattern velocity, the spike activity is increased during preferred-direction motion and decreased during null-direction motion. The V1 cell generally responds with lower peak activities to dynamic motion than to constant-velocity motion $(186 \pm 5.7$ and $240 \pm 8.3 \mathrm{~Hz}$, same V1 
cell) (compare Figs. 3A, 2A). As already shown previously (Fig. 2), the baseline activity of $\mathrm{V} 1$ increases after selective laser ablation of a VS2/3 cell (from $29 \pm 12$ to $84 \pm 11 \mathrm{~Hz}$ ) (Fig. $3 A$, dark gray line). Nevertheless, the spike activity modulations of V1 during dynamic motion stimulation appear to be relatively similar before and after ablation (Fig. 3A). Apart from slightly different absolute activity levels after ablation, the spike activity of V1 still follows the time course of pattern velocity. This is surprising because, after ablation of one input element, V1 has to read out the motion information from a smaller number of synaptic inputs.

To quantitatively assess the impact of VS2/3 ablation on the ability of V1 to encode the motion-velocity fluctuations, we applied a linear reconstruction technique (for details, see Materials and Methods). This approach is based on the correlation between instantaneous motion velocity and corresponding neural activity. It calculates a filter that, when convolved with the postsynaptic spike activity, minimizes the error between reconstructed and actual motion velocities. We used this technique to determine the coherence that indicates how well the different frequency components of the motion stimulus are transformed into the activities of V1. Coherence values of 1 indicate that the motion stimulus and the neural activity are related in a perfectly linear and noise-free way at the corresponding stimulus frequency. Coherence values below 1 can be attributed to nonlinearities or noise (or both) within the motion pathway.

Figure $3 B$ shows the averaged coherence values between the spike activity of four V1 cells and the eight dynamic motion sequences as well as the corresponding reconstruction filters (Fig. $3 C$ ) each before (black lines) and after (gray lines) ablation of a VS2/3 cell: before ablation, the coherence values are relatively high for frequencies below $8 \mathrm{~Hz}$ but decrease steeply for higher frequencies and reach 0 at $\sim 30 \mathrm{~Hz}$. This frequency dependence of motion-velocity coding is in accordance with a previous study (Warzecha et al., 2003). Ablation of a VS2/3 cell does not much change the coherence values determined for the same motion sequences. Hence, despite the elimination of one presynaptic cell and the increase in baseline activity of V1, the ability to represent motion velocity remains unaffected. This is also reflected by the similarity of the reconstructed velocity signals before (black line) and after (dark gray line; data from the same experiment as in Fig. $3 A$ ) ablation and the similar deviation from the original signal (Fig. 3D, gray line).

Thus, the encoding capacity of $\mathrm{V} 1$ does not change much as a consequence of ablating VS2/3. Whereas in some ablation experiments the coherence values for frequencies up to $10 \mathrm{~Hz}$ slightly decrease, they even increase in other experiments. To substantiate the validity of our coherence estimates, we performed control experiments with both a significantly larger dataset and a longer dynamic motion trace (for details, see Materials and Methods). These controls were performed on the basis of V1 responses without simultaneously ablating presynaptic cells, because the limited duration of stable recordings did not allow us to substantially increase the number of stimulus presentations or the duration of stimulus sequences in ablation experiments. The coherence analyses on the basis of $\mathrm{V} 1$ recordings with more stimulus presentations (Fig. 3E, gray line) and longer stimulus sequences (Fig. 3E, black line) yielded similar coherence values as obtained under our standard conditions. Thus, the analyses in our ablation experiments can be regarded as valid estimations of coherences in the entire frequency domain. This makes an underestimation of the coherences in our ablation experiments, which otherwise might have masked ablation-induced effects, very unlikely.

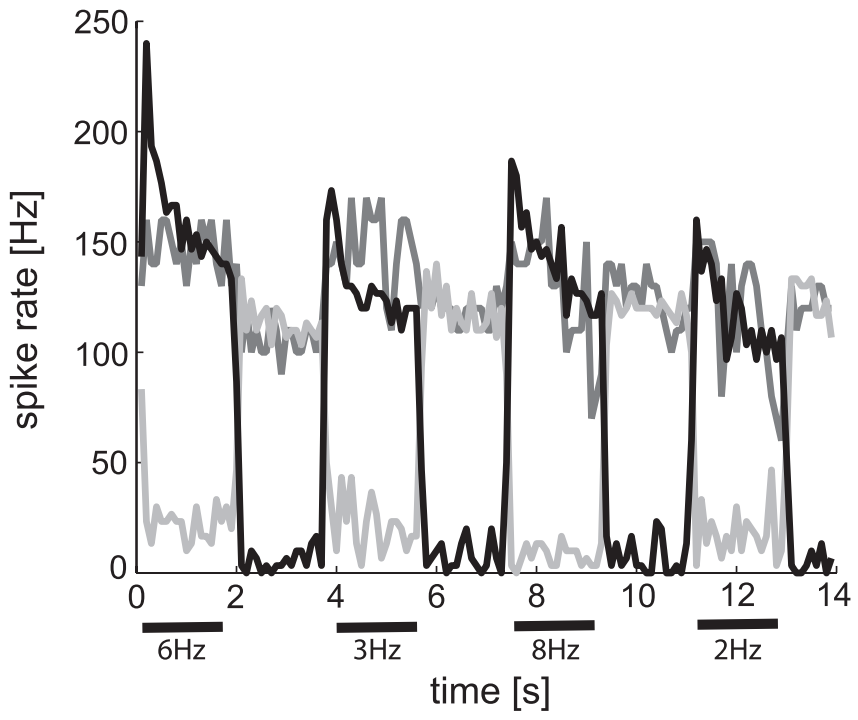

Figure 4. Impact of VS1 ablation on the response properties of V1 during constant-velocity motion stimulation. Mean spike frequencies before (black line), initially after ablation (dark gray line), and $\sim 5$ min after ablation (gray line). V1 spikes were counted in 100 ms time segments. Initially after ablation, a dramatic increase in baseline activity of V1 was observed, and motion-induced changes in activity were small. Later, the spike activity was reduced below its baseline activity during preferred-direction motion stimulation.

All in all, our results indicate that the neural circuit formed by VS cells and V1 is robust with respect to motion coding. Ablation of individual VS2/3 cells is associated with a shift of the operating range of $\mathrm{V} 1$ from its normal state to a more excited condition but leaves the capacity to encode motion velocity intact.

Finally, we applied a measure that calculates the information conveyed by neural responses without assuming a linear stimulus-response relationship (Brenner et al., 2000). We found similar results when we calculated the information content of the responses of $\mathrm{V} 1$ before and after ablation of VS2/3: the average information rate after ablation of a VS2/3 cell was $30 \pm 6$ compared with $35 \pm 4.5$ bits/s before ablation (mean $\pm \mathrm{SD} ; n=4$ ). Thus, the information transfer between the input VS cells and V1 does not change dramatically after eliminating one presynaptic input element.

These results suggest that, in addition to the shift in the operating range of the system, the transfer of motion information between input VS cells and V1 may bear considerable redundancy, such that loss of inputs may not result in severe functional consequences, at least on the transmission of motion-velocity fluctuations in the frequency range below $10 \mathrm{~Hz}$.

\section{Effect of VS1 cell ablation and of ablation of a VS cell with a lateral maximum sensitivity to downward motion}

The ablation of the VS1 cell affects the response properties of V1 more profoundly than ablation of VS2/3. As can be seen in the spike activity histograms in Figure 4 (dark gray line, spike activities evaluated within $100 \mathrm{~ms}$ time windows), the baseline activity of V1 strongly increases immediately after ablation of VS1. This increase in baseline activity is accompanied by a reduced increment of the motion-induced activities relative to the baseline activity. This effect, however, is unstable. A few minutes later, V1 appears to reverse its direction selectivity (Fig. 4, gray line): now preferred-direction motion reduces the spike activity below the elevated baseline activity. The seemingly paradoxical reduction in spike activity during preferred-direction motion might be attributable to sodium-channel inactivation during sustained above- 

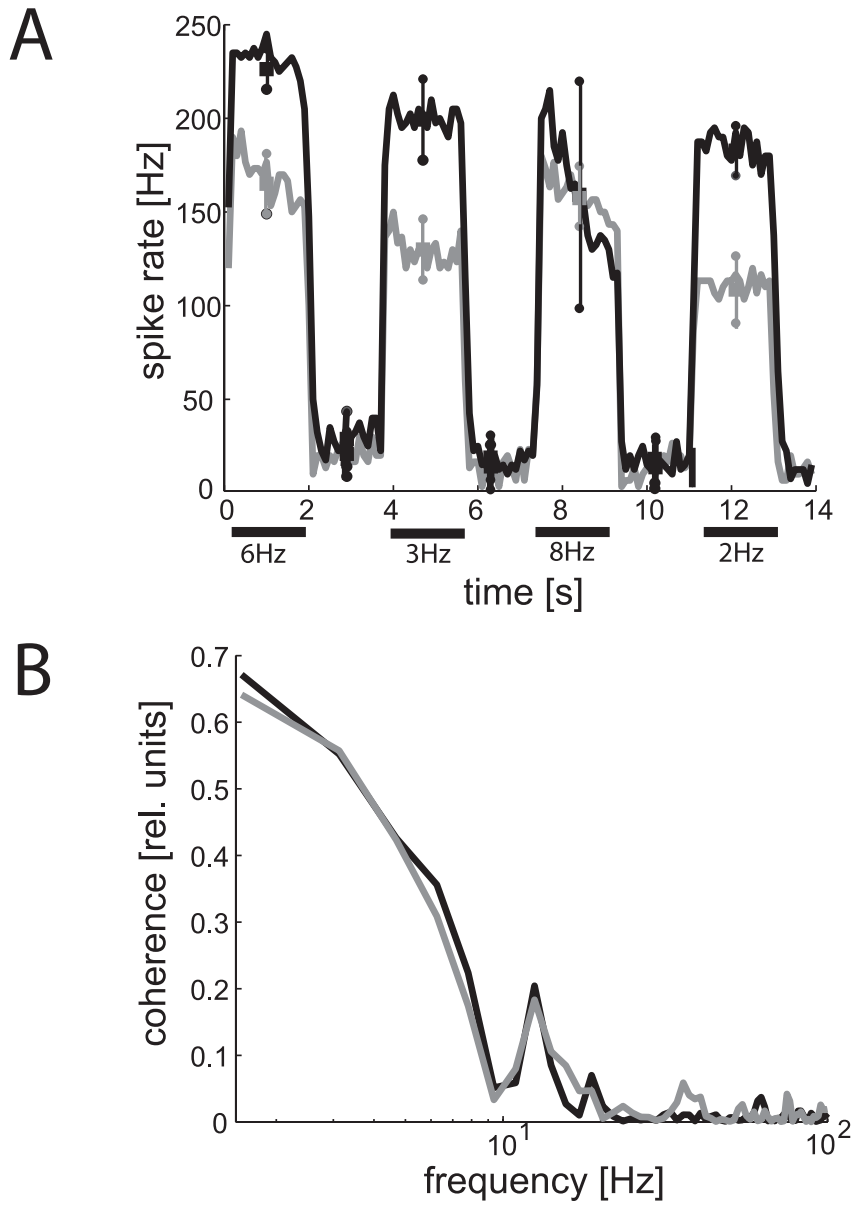

Figure 5. Response change of V1 after ablation of a presynaptic VS cell with a lateral maximum sensitivity to downward motion. $\boldsymbol{A}$, Spike frequency histograms (mean spike frequencies averaged over 4 stimulus repetitions; temporal resolution of $100 \mathrm{~ms}$ ) before (black line) and after (gray line) ablation. Squares and circles denote means and SDs of motion-induced and baseline activity of $\mathrm{V} 1$, respectively. Whereas the motion-induced response components are reduced, the baseline activity remains unchanged after ablation. $\boldsymbol{B}$, Coherence functions before (black line) and after (gray line) ablation. The corresponding coherence values did not change after ablation of a VS cell with a lateral receptive field.

threshold depolarization. Similar effects have been observed during current injection into graded-potential tangential cells with respect to the generation of spikelets (Hengstenberg, 1977). In two additional VS1 ablation experiments, we observed equally strong effects on V1 (data not shown): the ablation is initially followed by a marked increase in baseline activity of V1 and leads to a complete breakdown of the activity after a few minutes. Hence, ablation of VS1 has a much stronger impact on both the baseline activity and the motion-induced activities of V1 than ablation of VS2/3 (compare Figs. 2, 4).

In one ablation experiment, we ablated one of the VS cells, which are sensitive to downward motion in predominantly lateral parts of the visual field (VS4, VS5, or VS6). The spatial extent of the receptive field of V1 suggests weak input from at least one of these cells (Krapp et al., 2001). Figure 5A compares the spike activity histograms before (Fig. $5 A$, black line) and 20 min after (gray line) ablation: the motion-induced activities are reduced from, on average, $192 \pm 28$ to $140.5 \pm 25.8 \mathrm{~Hz}$. This reduction in stimulus-induced spike activity provides evidence for visual input from VS4 (VS5/6) to V1. In contrast to ablation of VS1-VS3, baseline activity of $\mathrm{V} 1$ does not change prominently (compare $21.3 \pm 7$ with $16.3 \pm 4 \mathrm{~Hz}$ ). When we compare coherence values before and after ablation, we find no prominent changes (Fig. $5 B)$. Similar to the results obtained after the ablation of a VS2/3 cell, the ability of $\mathrm{V} 1$ to encode time-dependent motion velocities is not affected by the elimination of synaptic motion inputs from a VS cell with a lateral receptive field.

\section{Discussion}

The VS-V1 circuit presents an excellent model system to study how convergent synaptic inputs are integrated to extract information from a time-varying stimulus in the presence of noise. The brain is confronted with similar tasks in other contexts, such as during somatosensory coding of rats (Higley and Contreras, 2003; Waters et al., 2003), during integration of light signals in the vertebrate and insect retina (de Ruyter van Steveninck and Laughlin, 1996; Field and Rieke, 2002), and during detection of stimulus direction in the mechanosensory cercal system of the cricket (Miller et al., 1991; Theunissen and Miller, 1991).

We analyzed the contribution of individual presynaptic VS cells to visual-motion coding of the postsynaptic V1 cell by laser ablation of single VS cells. VS ablation has two consequences. First, synaptic input normally contributing to the $\mathrm{V} 1$ responses is lost. Second, ablation leads to an increase in the baseline activity of V1. Nonetheless, encoding of the motion velocity of V1 is not degraded after ablation of VS2/3. This capacity suggests robust encoding of visual motion information in the analyzed neuronal circuit. In contrast, ablation of VS1 affects the motion responses of V1 more profoundly, which, in addition to a massive increase in baseline activity, may reverse its direction selectivity or even cease spiking.

\section{Neuronal wiring schemes explaining the ablation-induced activity changes}

Because the connections between V1 and its input elements are excitatory (Kurtz et al., 2001; Warzecha et al., 2003), the ablationinduced increases in baseline activity are likely attributable to an increase in excitation, induced by the depolarization of the ablated VS cell (Farrow et al., 2003). The limited transmitter pool size of chemical synapses (for review, see Sterling and Matthews, 2005) makes ongoing transmitter release over extended time periods highly improbable. In our experiments, however, the rise in baseline activity was stable for more than $1 \mathrm{~h}$. Therefore, current flow through electrical synapses between neighboring VS cells most likely play a role: the voltage difference between the depolarized membrane potential of the ablated cell and the membrane potential of the electrically connected cell is likely to induce tonic leakage currents through the gap junctions. In the following, we will discuss different synaptic connection schemes to explain the ablation-induced changes in the activity of V1.

Assuming only chemical synapses between VS cells and V1 (Fig. 6A), ablation of a VS cell might induce permanent current flow through electrical synapses into neighboring VS cells, depolarizing their membrane potential and indirectly exciting V1. This hypothesis is corroborated by the detection of electrical connections between neighboring VS cells by electrophysiological and dye-coupling studies (Haag and Borst, 2004, 2005) and by the existence of specializations typical of chemical synapses in VS terminals (Hausen et al., 1980). Conversely, ablation of VS1 had the strongest impact on V1. This would require the synaptic gain between VS2/3 and V1 to be stronger than that between the other VS cells and V1. Only in this case, current flow from VS1 into VS2/3 would affect V1 more than currents flow in the reverse direction after ablation of VS2/3. This is in contrast to the receptive field organization of $\mathrm{V} 1$, which suggests that $\mathrm{V} 1$ gets promi- 

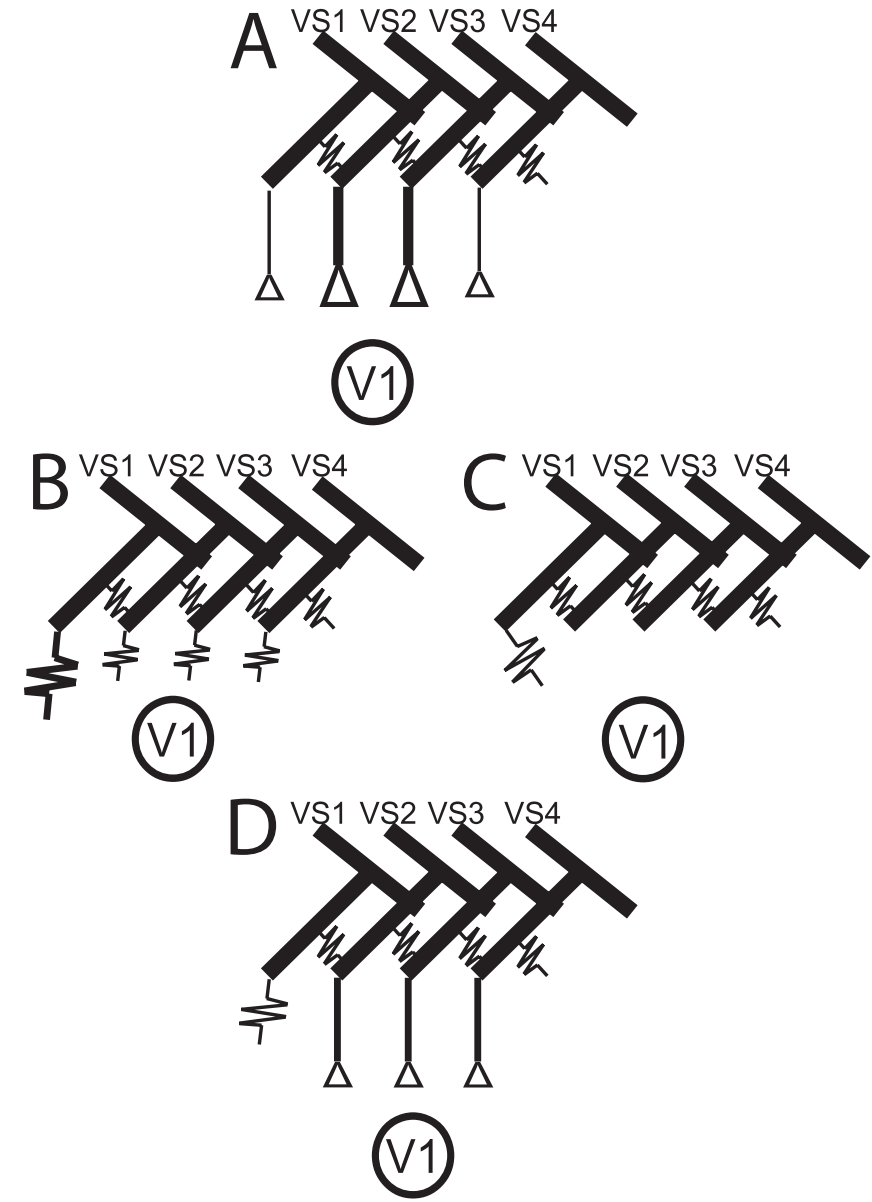

Figure 6. Different wiring schemes for the localization of chemical and/or electrical synapses between presynaptic VS cells and the postsynaptic V1, which might explain the observed ablation-induced changes in baseline and motion-induced activity of V1. Coupling of VS cells by electrical synapses was recently shown by Haag and Borst (2004). A, Chemical synapses between VS cells and V1 with synaptic gain being stronger between VS2/3 and V1 than between the other VS cells and V1. $\boldsymbol{B}$, In case of electrical synaptic connections within the neural VS-V1 circuit, the coupling strength between VS1 and V1 would have to be strongest to explain the results. C, VS1 forms the only direct coupling with V1 via electrical synapses. D, Electrical connections between VS1 and V1 and chemical synapses between the other presynaptic VS cells and $\mathrm{V} 1$ are in good agreement with the experimental findings.

nent input from VS1 (Krapp et al., 1998, 2001). Moreover, the electrical coupling strength between neighboring VS cells was found to be rather weak (Haag and Borst, 2004), suggesting that current flow from the ablated cell to its neighbor VS cell via electrical synapses would hardly be sufficient to drive the V1 cell up to its limit as was observed after VS1 ablation. Moreover, ablation of a more lateral sensitive VS cell did not induce any increase in baseline activity of V1 (Fig. 5A).

Alternatively, solely electrical synapses between V1 and its presynaptic VS cells (Fig. 6B) might exist and directly depolarize V1 after VS ablation. In this case, V1 should be coupled more strongly to VS1 than to the other VS cells, because VS1 ablation affects the activity of V1 most profoundly. However, the missing increase in baseline activity of V1 after ablation of a lateral VS cell cannot easily be reconciled with the assumption of purely electrical couplings between VS cells and V1.

In principle, our results agree with $\mathrm{V} 1$ receiving direct input via an electrical synapse exclusively from VS1 and only indirect input from other VS cells via their electrical coupling to VS1 (Fig. 6C). However, the weak electrical coupling strength between VS cells (Haag and Borst, 2004) can hardly explain the strong increase in V1 spike activity during current injection into VS2/3 (Kurtz et al., 2001; Warzecha et al., 2003).

Our experiments can be explained best by assuming electrical synapses between VS1 and V1 and chemical synapses between the other presynaptic VS cells and V1 (Fig. 6D). The dramatic increase in baseline activity after ablation of VS1 can then be attributed to a direct effect, whereas the comparatively weaker increase after ablation of VS2/3 would be an indirect effect attributable to electrical coupling between VS cells. The missing increase in baseline activity of V1 after ablation of a more lateral VS cell further corroborates the proposed wiring scheme: in this case, neither a direct effect nor a prominent indirect effect is expected because of the large electronic distance to VS1.

To further clarify the nature of VS-V1 synapses, we applied $\mathrm{Co}^{2+}$ locally to the input region of V1 (supplemental Fig. 2, available at www.jneurosci.org as supplemental material). $\mathrm{Co}^{2+}$, which is known to block chemical synaptic transmission (Alvarez-Leefmans et al., 1979), reduced the motion-induced responses of V1. However, the motion responses of V1 were not entirely blocked, consistent with our model in Figure $6 D$ with both chemical and electrical synapses. As an alternative to separate chemical and electrical synapses between individual VS neurons and V1, there might exist mixed chemical/electrical synapses as have been demonstrated in other areas of the fly brain (Strausfeld and Bassemir, 1983; Fayyazuddin and Dickinson, 1996).

Because it was shown previously that presynaptic rapid membrane potential depolarizations are reliably transmitted with a millisecond precision (Warzecha et al., 2003), it might be speculated that electrical connections function to transmit these fast signals, whereas the chemical synapses between VS cells and V1 seem to be adapted to convey graded membrane potential changes (Kurtz et al., 2001).

\section{Redundancies in synaptic connectivity may help making} integration of visual-motion information more robust

The presynaptic VS cells and V1 have similar receptive fields, preferred axes of self-motion, and a similar velocity tuning (Hengstenberg, 1982, Krapp et al., 1998, 2001; Karmeier et al., 2003). Nonetheless, given cell-intrinsic noise, pooling the outputs of several neurons with similar functional properties may lead to a more reliable representation of motion information than with single elements missing. Contrary to this expectation, $\mathrm{V} 1$ does not represent time-dependent velocity fluctuations less reliably after ablation of most presynaptic elements. Only did ablation of VS1 radically affect the baseline activity of VS1, such that it was impossible to determine coherence functions.

The ablation of a presynaptic VS cell did not only eliminate an element providing information but additionally induced an increase of the baseline activity of V1. The latter effect may additionally decrease the input resistance and, thus, the gain of V1. Thus, unspecific excitation may be expected to present an additional constraint to the ability of V1 to gather accurate motion information from its residual input. Conversely, the elevated baseline activity may improve the ability of V1 to encode nulldirection motion, thus compensating its negative effects to some extent.

Because elimination of single presynaptic elements may remain without negative functional consequences for the postsynaptic neuron, presynaptic information appears to bear redundancies. Synaptic integration by V1 seems to be organized in such a way that certain stimulus features are robustly extracted even in the presence of reduced presynaptic activity. This is in accor- 
dance with a previous study on population coding of self-motion in the fly: although the VS population consists of 10 neurons, theoretically no more than two of them are sufficient to encode any horizontal self-rotation axis (Karmeier et al., 2005).

An overrepresentation of sensory inputs is found in many neural systems (Reich et al., 2001; Puchalla et al., 2005) and might lead to advantages under more demanding stimulus conditions, such as low contrast levels or with smaller or more heterogeneous stimuli. We cannot exclude that extraction of stimulus features other than motion velocity, for example the axis of self-rotation of the animal, or computations that rely on the comparison of the activity of the V1 cells of both halves of the visual system are more critically affected by VS ablation. In a previous study, the comparison of membrane potential fluctuations of VS cells with V1 spike activities during white-noise motion stimulation revealed that spikes in V1 are coupled with high temporal precision to fast transients in the VS voltage signal (Warzecha et al., 2003). Because of common inputs and electrical coupling between VS cells, such voltage transients might be synchronously elicited in neighboring VS cells. Ablation of individual VS cells may therefore reduce the coupling between VS and V1 on a fast timescale. This aspect could only be addressed by performing VS-V1 simultaneous recordings after ablation of one of the other VS cells. It is, however, unclear whether coupling of VS and V1 on such a fast timescale has a major functional significance, because the coding of velocity fluctuations in the visual system is limited to much slower timescales (Warzecha et al., 1998, 2003).

\section{References}

Alvarez-Leefmans FJ, De Santis A, Miledi R (1979) Effects of some divalent cations on synaptic transmission in frog spinal neurones. J Physiol (Lond) 294:387-406.

Borst A, Haag J (2002) Neural networks in the cockpit of the fly. J Comp Physiol A Neuroethol Sens Neural Behav Physiol 188:419-437.

Borst A, Theunissen FE (1999) Information theory and neural coding. Nat Neurosci 2:947-957.

Brenner N, Strong SP, Koberle R, Bialek W, de Ruyter van Steveninck RR (2000) Synergy in a neural code. Neural Comput 12:1531-1552.

de Ruyter van Steveninck RR, Laughlin SB (1996) The rate of information transfer at graded-potential synapses. Nature 379:642-645.

Dürr V, Egelhaaf M (1999) In vivo calcium accumulation in presynaptic and postsynaptic dendrites of visual interneurons. J Neurophysiol 82:3327-3338.

Egelhaaf M, Borst A (1993) A look into the cockpit of the fly: visual orientation, algorithms, and identified neurons. J Neurosci 13:4563-4574.

Egelhaaf M, Kern R, Krapp HG, Kretzberg J, Kurtz R, Warzecha A-K (2002) Neural encoding of behaviourally relevant visual-motion information in the fly. Trends Neurosci 25:96-102.

Farrow K, Haag J, Borst A (2003) Input organization of multifunctional motion-sensitive neurons in the blowfly. J Neurosci 23:9805-9811.

Farrow K, Borst A, Haag J (2005) Sharing receptive fields with your neighbors: tuning the vertical system cells to wide field motion. J Neurosci 25:3985-3993.

Fayyazuddin A, Dickinson MH (1996) Haltere afferents provide direct, electrotonic input to a steering motor neuron in the blowfly, Calliphora. J Neurosci 16:5225-5232.

Field GD, Rieke F (2002) Nonlinear signal transfer from mouse rods to bipolar cells and implications for visual sensitivity. Neuron 34:773-785.

Haag J, Borst A (2004) Neural mechanism underlying complex receptive field properties of motion-sensitive interneurons. Nat Neurosci 7:628-634

Haag J, Borst A (2005) Dye-coupling visualizes networks of large-field motion-sensitive neurons in the fly. J Comp Physiol A Neuroethol Sens Neural Behav Physiol Neuroethol Sens Neural Behav Physiol 191:445-454.

Hausen K, Egelhaaf M (1989) Neural mechanisms of visual course control in insects. In: Facets of vision (Stavenga DG, Hardie RC, eds), pp 391424. Berlin: Springer.

Hausen K, Wolburg-Buchholz W, Ribi WA (1980) The synaptic organization of visual interneurons in the lobula complex of flies. A light and electron microscopical study using silver- intensified cobaltimpregnations. Cell Tissue Res 208:371-387.

Hengstenberg R (1977) Spike responses of "non-spiking” visual interneurone. Nature 270:338-340.

Hengstenberg R (1982) Common visual response properties of giant vertical cells in the lobula plate of the blowfly Calliphora. J Comp Physiol A Neuroethol Sens Neural Behav Physiol 149:179-193.

Hengstenberg R, Hausen K, Hengstenberg B (1982) The number and structure of giant vertical cells (VS) in the lobula plate of the blowfly Calliphora erythrocephala. J Comp Physiol A Neuroethol Sens Neural Behav Physiol 149:163-177.

Higley MJ, Contreras D (2003) Nonlinear integration of sensory responses in the rat barrel cortex: an intracellular study in vivo. J Neurosci 23:10190-10200.

Juusola M, Uusitalo RO, Weckstrom M (1995) Transfer of graded potentials at the photoreceptor-interneuron synapse. J Gen Physiol 105:117-148.

Karmeier K, Krapp HG, Egelhaaf M (2003) Robustness of the tuning of fly visual interneurons to the tuning to rotatory optic flow. J Neurophysiol 90:1626-1634.

Karmeier K, Krapp HG, Egelhaaf M (2005) Population coding of self-motion: applying bayesian analysis to population of visual interneurons in the fly. J Neurophysiol 94:2182-2194.

Krapp HG, Hengstenberg B, Hengstenberg R (1998) Dendritic structure and receptive-field organization of optic flow processing interneurons in the fly. J Neurophysiol 79:1902-1917.

Krapp HG, Hengstenberg R, Egelhaaf M (2001) Binocular contributions to optic flow processing in the fly visual system. J Neurophysiol 85:724-734.

Kurtz R (2004) $\mathrm{Ca}^{2+}$ clearance in visual motion-sensitive neurons of the fly studied in vivo by sensory stimulation and UV photolysis of caged $\mathrm{Ca}^{2+}$. J Neurophysiol 92:458-467.

Kurtz R, Warzecha AK, Egelhaaf M (2001) Transfer of visual motion information via graded synapses operates linearly in the natural activity range. J Neurosci 21:6957-6966.

Miller JP, Jacobs GA, Theunissen FE (1991) Representation of sensory information in the cricket cercal sensory system. I. Response properties of the primary interneurons. J Neurophysiol 66:1680-1689.

Puchalla LJ, Schneidman E, Harris AR, Berry JM (2005) Redundancy in the population code of the retina. Neuron 46: 493-504.

Reich SD, Mechler F, Victor DJ (2001) Independent and redundant information in nearby cortical neurons. Science 294:2566-2568.

Sterling P, Matthews G (2005) Structure and function of ribbon synapses. Trends Neurosci 28:20-29.

Strausfeld NJ, Bassemir UK (1983) Cobalt-coupled neurons of a giant fibre system in Diptera. J Neurocytol 12:971-991.

Theunissen FE, Miller JP (1991) Representation of sensory information in the cricket cercal sensory system. II. Information theoretic calculation of system accuracy and optimal tuning-curve widths of four primary interneurons. J Neurophysiol 66:1690-1703.

Warzecha AK, Kretzberg J, Egelhaaf M (1998) Temporal precision of the encoding of motion information by visual interneurons. Curr Biol 8:359368.

Warzecha AK, Kurtz R, Egelhaaf M (2003) Synaptic transfer of dynamic motion information between identified neurons in the visual system of the blowfly. Neuroscience 119:1103-1112.

Waters J, Larkum M, Sakmann B, Helmchen F (2003) Supralinear $\mathrm{Ca}^{2+}$ influx into dendritic tufts of layer $2 / 3$ neocortical pyramidal neurons in vitro and in vivo. J Neurosci 23:8558-8567. 